\title{
The impossibility of social evaluations of infinite streams with strict inequality aversion
}

\author{
José Carlos R. Alcantud
}

Received: 11 March 2013 / Accepted: 13 March 2013 / Published online: 30 March 2013

(C) SAET 2013

\begin{abstract}
We are concerned with the problem of aggregating infinite utility streams and the possible adoption of consequentialist equity principles. We find a virtually universal incompatibility between the Basu-Mitra approach (that advocates for social welfare functions and renounces continuity assumptions) and postulates that capture various forms of strict preference for a reduction in inequality like the Strong Equity Principle, the Pigou-Dalton Transfer principle, or Altruistic Equity. We also prove that the Hara-Shinotsuka-Suzumura-Xu impossibility for semicontinuous social welfare relations remains under the latter distributional postulate.
\end{abstract}

Keywords Social welfare function · Inequality aversion · Pigou-Dalton transfer principle $\cdot$ Monotonicity

JEL Classification D63 $\cdot$ D71 $\cdot$ D90

\section{Introduction}

This paper is primarily concerned with the problem of aggregating infinite utility streams and the possible adoption of distributive equity principles. We prove that there is a fundamental incompatibility between salient postulates of strict inequality aversion like the Strong Equity Principle (Bossert et al. 2007), the Pigou-Dalton Transfer principle (Sakai 2006; Bossert et al. 2007), the Lorenz Domination principle

J. C. R. Alcantud ( $\bigotimes)$

Campus Unamuno. Edificio FES, Universidad de Salamanca,

37007 Salamanca, Spain

e-mail: jcr@usal.es

URL:http://diarium.usal.es/jcr 
(Hara et al. 2008), or Altruistic Equity (Hara et al. 2006; Sakamoto 2012), and the Basu-Mitra approach that uses social welfare functions (SWFs) and renounces continuity. If we abandon the use of utilities, we complement Sakai (2006) and Hara et al. (2008) to conclude that those postulates are incompatible with semicontinuous preferences too.

As Sakai (2006) has put it, there are two ethical considerations that capture the concept of intergenerational equity: inequality aversion and equality in evaluating allocations. The latter is in the utilitarian tradition à-la-Sidgwick and Diamond, and requires that the welfare orderings should not be biased against any generation. In formal terms, it appeals to anonymity axioms that impose the impartial treatment of all generations. The former has received much attention in recent years. The aforementioned distributional axioms have been introduced in the literature on intergenerational justice to explore the implications of such ethical standpoint; thus, they have the common spirit of expressing a strict preference for distributions of utilities among generations that reduce inequality in various forms.

Irrespective of the egalitarian position that is adopted, the essential shortfall of the approach by numerical evaluations or SWFs in this context has been brought to the fore by a number of contributions. Either, if one requests anonymity-type properties (Basu and Mitra 2003; Crespo et al. 2009), the very mild Hammond Equity for the Future (Banerjee 2006), or variations of other consequentialist principles of aversion to inequality (Alcantud 2010, 2012; Sakamoto 2012), relaxed-but not universally acceptable-versions of the Pareto principle like strong Pareto, weak dominance, or weak Pareto, lead to incompatibility under different specifications of the domain of utility sequences. Here we go further and prove that assuming only the uncontroversial monotonicity for nearly unrestricted sets of admissible utilities, SWFs must contradict the ethos of the Pigou-Dalton transfer principle at the level of a especially plausible generalization named Altruistic Equity. Other distributional axioms implying Pigou-Dalton under monotonicity, like the Strong Equity Principle or the Lorenz domination principle, turn out to be incompatible with the Basu-Mitra approach.

To illustrate further the difficulty of implementing strict aversion to inequality, we prove that acyclic evaluations which are semicontinuous with respect to the sup topology cannot verify Altruistic Equity. This is a variant of previous results in Hara et al. (2008).

This paper is organized as follows. Section 2 introduces the setting and axioms. Section 3 proves our main result. Section 4 concludes and briefly reports on related literature, including our impossibility result for acyclic relations.

\section{Notation and definitions}

Let $\mathbf{X} \subseteq \mathbb{R}^{\mathbb{N}}$ represent a domain of infinite-horizon utility streams. For simplicity, we assume $\mathbf{X}=Y^{\mathbb{N}}$ and say that $Y$ is the set of feasible or admissible utilities. We adopt the standard notation for infinite streams: $\mathbf{x}=\left(x_{1}, \ldots, x_{n}, \ldots \ldots\right) \in \mathbf{X}$. We write $\mathbf{x} \geqslant \mathbf{y}$ if $x_{i} \geqslant y_{i}$ for each $i=1,2, \ldots ; \mathbf{x} \gg \mathbf{y}$ if $x_{i}>y_{i}$ for each $i=1,2, \ldots$; and $\mathbf{x}>\mathbf{y}$ if $\mathbf{x} \geqslant \mathbf{y}$ and $\mathbf{x} \neq \mathbf{y}$. 
Social welfare relations are binary relations on $\mathbf{X}$. They are interpreted as normative welfare criteria on the domain $\mathbf{X}$. A social welfare function (SWF) is a function $\mathbf{W}: \mathbf{X} \longrightarrow \mathbb{R}$, also regarded as a representable social welfare relation. The analysis of intergenerational aggregation by means of SWFs is usually called the Basu-Mitra approach, which does not suppose a rejection of binary relations in the evaluation of utility streams.

Let $\mathbf{W}$ be a SWF. We proceed to recall some efficiency axioms that we use along the paper. First, we present the most standard version of the Pareto axiom. It is the very demanding principle that improving the allocation of at least one generation should increase the social evaluation:

Axiom SP (Strong Pareto). If $\mathbf{x}, \mathbf{y} \in \mathbf{X}, \mathbf{x}>\mathbf{y}$, then $\mathbf{W}(\mathbf{x})>\mathbf{W}(\mathbf{y})$.

The next efficiency axioms are all implied by Strong Pareto.

Axiom MON (Monotonicity). If $\mathbf{x}, \mathbf{y} \in \mathbf{X}, \mathbf{x} \geqslant \mathbf{y}$, then $\mathbf{W}(\mathbf{x}) \geqslant \mathbf{W}(\mathbf{y})$.

$\mathrm{MON}$ is regarded as a necessary condition for efficiency; thus, our results refer to monotonic SWFs. Another fairly justifiable weakening of SP follows:

Axiom WP (Weak Pareto). If $\mathbf{x}, \mathbf{y} \in \mathbf{X}, \mathbf{x} \gg \mathbf{y}$ then $\mathbf{W}(\mathbf{x})>\mathbf{W}(\mathbf{y})$.

An independent weaker version of Strong Pareto is Weak Dominance:

Axiom WD (Weak Dominance). If $\mathbf{x}, \mathbf{y} \in \mathbf{X}$ and there is $j \in \mathbb{N}$ such that $x_{j}>y_{j}$, and $x_{i}=y_{i}$ for all $i \neq j$, then $\mathbf{W}(\mathbf{x})>\mathbf{W}(\mathbf{y})$.

Now we recall some axioms that intend to prioritize more egalitarian allocations by expressing a strict preference for certain distributions of utilities among generations. The first distributional axiom is based on a fundamental postulate in the literature on income inequality measurement that was introduced by Pigou (1912) and Dalton (1920). Adapted versions of the principle have been explored in the context of social welfare orderings on allocations of various types to finite societies (cf., Kolm 1977; Moulin 1991; Fleurbaey and Michel 2003; Fleurbaey and Tungodden 2010, among others). In our context, it is a notion of inequality aversion in a cardinal vein that has been imported by Bossert et al. (2007) — under the name strict transfer principle — and Sakai (2006). It claims that a transfer of utility from a richer generation to a poorer generation must be socially beneficial provided that their relative positions do not change and that there is no cost of transfer. Formally:

Axiom PDT (Pigou-Dalton transfer principle) If $\mathbf{x}, \mathbf{y} \in \mathbf{X}$, there is $\varepsilon>0$ with $y_{j}=x_{j}-\varepsilon \geqslant y_{k}=x_{k}+\varepsilon$ for some $j, k \in \mathbb{N}$, and $x_{t}=y_{t}$ when $j \neq t \neq k$, then $\mathbf{W}(\mathbf{y})>\mathbf{W}(\mathbf{x})^{1}$.

A reinforced form of both PDT and the classical Hammond Equity postulate is the following axiom (Bossert et al. 2007):

Axiom SEP (Strict Equity Principle) If $\mathbf{x}, \mathbf{y} \in \mathbf{X}, x_{j}>y_{j} \geqslant y_{k}>x_{k}$ for some $j, k \in \mathbb{N}$, and $x_{t}=y_{t}$ when $j \neq t \neq k$, then $\mathbf{W}(\mathbf{y})>\mathbf{W}(\mathbf{x})$.

Under SEP, any utility sacrifice made by a richer generation that is rewarded by any utility gain by a poorer generation is socially beneficial when their relative positions do not change. Furthermore, the following restricted form of axiom SEP was considered by Hara et al. (2006):

\footnotetext{
1 Bossert et al. (2007) use an equivalent expression: If $\mathbf{x}, \mathbf{y} \in \mathbf{X}$ verify $x_{j}>y_{j} \geqslant y_{k}>x_{k}$ and $x_{j}+x_{k}=y_{j}+y_{k}$ for some $j, k \in \mathbb{N}$, and $x_{t}=y_{t}$ when $j \neq t \neq k$, then $\mathbf{W}(\mathbf{y})>\mathbf{W}(\mathbf{x})$.
} 
Axiom AE (Altruistic Equity) If $\mathbf{x}, \mathbf{y} \in \mathbf{X}$, there are $\varepsilon>\delta>0$ with $y_{j}=x_{j}-\delta \geqslant$ $y_{k}=x_{k}+\varepsilon$ for some $j, k \in \mathbb{N}$, and $x_{t}=y_{t}$ when $j \neq t \neq k$, then $\mathbf{W}(\mathbf{y})>\mathbf{W}(\mathbf{x})^{2}$.

We are especially interested in such relaxed version of axiom SEP because it suffices to prove our main impossibility theorem. Compared to the distributional rationales behind PDT and SEP, the ethical principle under AE is less demanding: It only claims that reductions in welfare for the rich that are accompanied by increases to the poor must be socially preferred when the gain of the poor is greater than the loss of the rich. Monotonic SWFs that verify MON/WD and PDT must verify AE (see Hara et al. 2006 for a related fact).

Finally, the Anonymity axiom (Axiom AN) claims that any finite permutation of a utility stream produces a socially indifferent utility stream.

\section{Pigou-Dalton, Altruistic Equity, and the existence of monotonic SWFs}

Alcantud (2010) (Proposition 5) proves that PDT and AN can be combined with WD under representability of the social evaluation. We proceed to show that this fact does not reconcile PDT with the Basu-Mitra approach: under extremely weak technical assumptions on the structure of the set of feasible utilities, every monotonic SWF must contradict the weaker AE.

Before stating our impossibility theorem, let us observe that PDT and AE hold vacuously unless $\mathbf{X}$ has some specific configuration. Since $\mathbf{X}=Y^{\mathbb{N}}$, in order for PDT, resp., AE, to impose real restrictions on the evaluations, the following must be true: There are $a, b, c \in Y$ such that $b-c=a-b>0$, resp., $b-c>a-b>0$. Therefore, to state our impossibility theorem with as weak as possible requirements, not only we need to refer to the cardinality or the ordinal properties of the set of feasible utilities but also to its intrinsic specification. Here we separate from other related antecedents when studying SWFs on domains of utility streams with the form $\mathbf{X}=Y^{\mathbb{N}}$. For example, in such context, the following facts are known. Basu and Mitra (2003) proves that AN and SP are incompatible as long as $Y$ has at least two different elements. Theorem 2 in Alcantud and García-Sanz (2013) assures that the Hammond Equity axiom and SP are incompatible as long as $Y$ has at least four different elements. Furthermore, Dubey and Mitra (2011) characterized the restrictions on $Y$ for which $\mathrm{AN}$ and WP are compatible. These are precisely the sets $Y$ that do not contain any set of the order-type of the set of integer numbers.

Now we are in a position to state our main result.

Theorem 1 Suppose there are $a, b, c, d \in Y \subseteq \mathbb{R}$ with $c-d>b-c>a-b>0$. Then there are no SWFs on $X=Y^{\mathbb{N}}$ that verify MON and $A E$.

Proof We use a standard construction to produce a suitable uncountable collection $\left\{E_{i}\right\}_{i \in I}$ of infinite subsets of $\mathbb{N}$. We request that $\forall j, i \in I\left[j<i \Rightarrow E_{j} \subsetneq\right.$

\footnotetext{
${ }^{2}$ Similarly one can define social welfare relations that verify AE, and we appeal to such concept in Proposition 1 below. Sakamoto (2012) uses the term Altruistic Equity-1 or AE-1 to refer to our Axiom AE. $\mathrm{He}$ also uses another variant of PDT that is called Altruistic Equity-2 or AE-2. Because we are maintaining the basic principle of monotonicity throughout, and AE-2 and SEP are equivalent under MON, we do not need to refer to $\mathrm{AE}-2$ here.
} 
$E_{i}$ and $E_{i}-E_{j}$ is infinite ], and $\{1,2\} \subseteq E_{i}$ for every $i \in I$. To justify that such collection exists, we take $\left\{r_{1}, r_{2}, \ldots\right\}$ an enumeration of the rational numbers in $I=(0,1)$, set $E^{\prime}(i)=\left\{n \in \mathbb{N}: r_{n}<i\right\}$ for each $i \in I$, and then $E(i)=E^{\prime}(i) \cup\{1,2\}$.

With each $i \in I$, let us associate two streams $r(i)$ and $l(i)$ as follows:

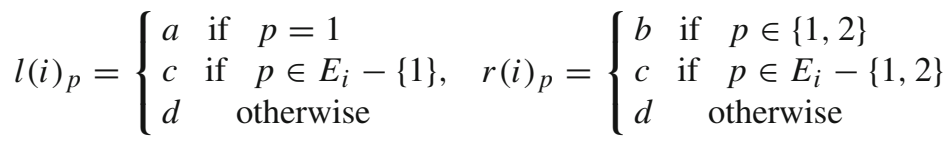

By AE, the open interval $(\mathbf{W}(l(i)), \mathbf{W}(r(i)))$ is not empty: When passing from $l(i)$ to $r(i)$, generation 1 loses $a-b$, while generation 2 gains $b-c>a-b$, and both generations have the same endowment at $r(i)$, namely $b$.

We intend to check that $j<i$ implies $\mathbf{W}(l(i))>\mathbf{W}(r(j))$, an absurd (an uncountable number of distinct rational numbers would be obtained). Select $k \in E_{i}-E_{j}$, thus $1 \neq k \neq 2$. We make use of an intermediate stream, namely

$$
\mathbf{z} \in \mathbf{X} \text { such that } z_{p}= \begin{cases}a & \text { if } p=1 \\ b & \text { if } p=2 \\ c & \text { if } p \in E_{i}-\{1,2, k\} \\ d & \text { otherwise }\end{cases}
$$

AE ensues $\mathbf{W}(l(i))>\mathbf{W}(\mathbf{z})$ : when passing from $\mathbf{z}$ to $l(i)$, generation 2 loses $b-c$, generation $k$ gains $c-d>b-c$, and both generations have endowment $c$ at $l(i)$. Now MON implies $\mathbf{W}(\mathbf{z}) \geqslant \mathbf{W}(r(j))$ and the thesis follows.

Obviously Theorem 1 conveys similar incompatibilities for distributional axioms implying AE under MON, like SEP, PDT, or the Lorenz domination principle (LD) which is stronger than PDT (Hara et al. 2008). Its assumption on the form of $Y$ is met by the most usual requirements. Therefore,

Corollary 1 If $\mathbb{N} \subseteq Y$ or $Y=[0,1]$ (or more generally, if $Y$ meets the conditions of Theorem 1), then there are no SWFs on $X=Y^{\mathbb{N}}$ that verify MON and SEP, resp., $P D T, L D, A E^{3}$.

\section{Conclusion and related literature}

Theorems 1 and 2 in Bossert et al. (2007) prove that both PDT and SEP are compatible with orderings on $\mathbb{R}^{\mathbb{N}}$ that verify SP and AN. Nevertheless, the literature on egalitarianism in the evaluation of infinite streams of utilities has provided evidences that the Pigou-Dalton transfer principle, as well as the Lorenz domination principle, conflict with weak forms of continuity and rationality even in the absence of Paretian restrictions (Sakai 2006; Hara et al. 2008, Theorems 1, 2). More precisely, Hara et al. prove that there exists no social evaluation $P$ satisfying LD, resp., PDT and acyclicity, and upper or lower semicontinuity with respect to the sup topology (each of which

\footnotetext{
3 Our proof of Theorem 1 permits to check that MON and PDT are incompatible when there exist $a, b, c, d \in$ $Y \subseteq \mathbb{R}$ such that $c-d=b-c=a-b>0$.
} 
is implied by Diamond's continuity $)^{4}$. We call this statement the Hara-ShinotsukaSuzumura-Xu (HSSS) impossibility theorem. Theorem 1 compares to this result in that without the appeal to any controversial form of the Paretian axiom but renouncing topological continuity assumptions, a principle that relaxes the Pigou-Dalton transfer principle into an ethically more acceptable form (namely, Altruistic Equity) is incompatible with a numerical evaluation of the utility streams for virtually unrestricted domains of streams.

On this background, it seems natural to study if appealing to the Altruistic Equity ethics instead makes the HSSS impossibility vanish. Proposition 1 below is a direct variation of their reasoning and concludes in the negative.

Proposition 1 No acyclic social evaluation $P$ on $\boldsymbol{X}=[0,1]^{\mathbb{N}}$ verifies $A E$ and upper or lower semicontinuity with respect to the sup topology.

Proof To prove the upper semicontinuity case, we replicate the argument in (Hara et al. 2008, Theorem 1), see also (Sakai 2006, Lemma 6). Thus, we just sketch our modified proof by stressing its prime elements. The case of lower semicontinuity is a direct variation.

Assume that $P$ verifies AE and upper semicontinuity with respect to the sup topology. We proceed to obtain $P$ cycles. By $P^{\infty}$ we denote the transitive closure of $P$, i.e., $\mathbf{x} P^{\infty} \mathbf{y}$ means that there are $n \in \mathbb{N}$ and $\mathbf{x}_{0}=\mathbf{x}, \mathbf{x}_{1}, \ldots, \mathbf{x}_{n}=\mathbf{y}$ in $\mathbf{X}$ such that $\mathbf{x}_{0} P \mathbf{x}_{1} P \ldots P \mathbf{x}_{n}$.

Let $\mathbf{y}^{0}=(0,1,0,1,0, \ldots)$ and, for each $n \in \mathbb{N}$, let

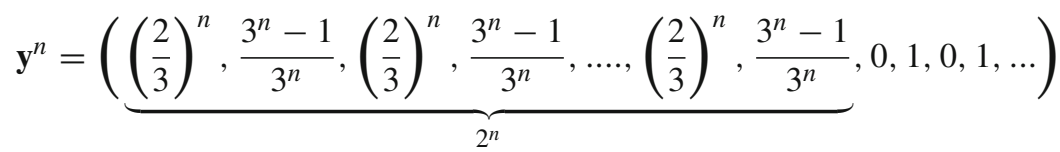

Then AE implies $\mathbf{y}^{1}=\left(\frac{2}{3}, \frac{2}{3}, 0,1,0,1, \ldots\right) P \mathbf{y}^{0}$, and also $\mathbf{y}^{n+1} P^{\infty} \mathbf{y}^{n}$ (thus $\mathbf{y}^{n+1} P^{\infty} \mathbf{y}^{1}$ ) for $n=1,2, \ldots$, since $\mathbf{y}^{n+1}$ can be obtained from $\mathbf{y}^{n}$ as follows:

1. Generations $1,3, . ., 2^{n}-1$ are assigned $\left(\frac{2}{3}\right)^{n}$ under $\mathbf{y}^{n}$. They lose one-third of that amount and generations $2^{n}+1,2^{n}+3, \ldots, 2^{n+1}-1$ that receive 0 under $\mathbf{y}^{n}$, gain twice as much as that (i.e., two-thirds of $\left.\left(\frac{2}{3}\right)^{n}\right)$. Under $\mathbf{y}^{n+1}$, their allocations are the same.

2. Generations $2,4, \ldots, 2^{n}$ are assigned $\frac{3^{n}-1}{3^{n}}=1-\frac{1}{3^{n}}$ under $\mathbf{y}^{n}$. Generations $2^{n}+$ $2,2^{n}+4, \ldots, 2^{n+1}$ are richer under $\mathbf{y}^{n}$ because they receive 1 . The difference in their endowments is $\frac{1}{3^{n}}$. The richer generations lose one-third of that difference, while the poorer generations gain two-thirds of it. Under $\mathbf{y}^{n+1}$, their allocations are the same.

Because $\left\|\mathbf{y}^{n+1}-\mathbf{y}^{0}\right\|_{\infty}=\left(\frac{2}{3}\right)^{n}$, the sequence $\left\{y^{n}\right\}_{n}$ converges to $\mathbf{y}^{0}$ in the sup topology. Now the fact $\mathbf{y}^{1} P \mathbf{y}^{0}$ and upper semicontinuity entail the existence of a cycle of $P$ involving $\mathbf{y}^{1}, \ldots, \mathbf{y}^{n}$ for every sufficiently large $n$.

\footnotetext{
4 The sup topology derives from the sup norm || $\mathbf{x} \|_{\infty}=\sup _{n}\left|x_{n}\right|$. Upper (resp., lower) semicontinuity with respect to the sup topology means that the lower (resp., upper) contour set of $P$ at any $\mathbf{x} \in \mathbf{X}$, i.e., $\{\mathbf{y} \in \mathbf{X}: \mathbf{x} P \mathbf{y}\}$ (resp., $\{\mathbf{y} \in \mathbf{X}: \mathbf{y} P \mathbf{x}\}$ ), is open in the sup topology on $\mathbf{X}$.
} 
The arguments above concur on the difficulty of implementing the ethos of strict preference for a reduction in inequality in the intergenerational welfare analysis. A possible route of escape that captures the rationale that inequality among generations should not be promoted, is expressed by just denying strict preference for certain rises in inequality. However, the literature has already provided some arguments against the feasibility of this programme for evaluations blending either continuity or representability. For example, Asheim et al. (2012, Propositions 3 and 4) prove that the weak versions of PDT/LD that arise are in conflict with very mild efficiency under a restricted version of continuity with respect to the sup topology. Hara et al. (2008, Proposition 2) states that such weakened version of LD is incompatible with WP and upper semicontinuity with respect to the sup topology, and that so is the weakened PDT under negative transitivity of the social welfare relation. As to representable criteria on $\mathbf{X}=[0,1]^{\mathbb{N}}$, Banerjee (2006) provides a negative answer for the very mild Hammond Equity for the Future assumption under WD, Alcantud and García-Sanz (2013) does the same for the Hammond Equilty postulate under a mild restricted version of WD; Alcantud (2012) proves that Hammond Equity is incompatible with WP, and Sakamoto (2012) proves that both PDT and AE are incompatible with WP (see also Alcantud 2012).

We complete this overview with the distinction between the existence of criteria with nice properties and the problem of explicitly describing one such criterion. In this regard, a conjecture by Fleurbaey and Michel (2003) about the constructibility of fair evaluations of infinite utility streams has received answers like Zame (2007), Lauwers (2010), or Dubey (2011).

Acknowledgments This research has been supported by the Spanish Ministerio de Economía y Competitividad under Project ECO2012-31933. The author wishes to thank the participants at the XXXVI Meeting of the Spanish Economic Association (SAEe) for helpful comments.

\section{References}

Alcantud, J.C.R.: The compromise efficiency vs. egalitarianism among generations with an infinite horizon. MPRA Paper No. 22467. http://mpra.ub.uni-muenchen.de/22467/ (2010)

Alcantud, J.C.R.: Inequality averse criteria for evaluating infinite utility streams: the impossibility of Weak Pareto. J. Econ. Theory 147, 353-363 (2012)

Alcantud, J.C.R., García-Sanz, M.D.: Evaluations of infinite utility streams: Pareto-efficient and egalitarian axiomatics. Metroeconomica. (2013). doi:10.1111/meca.12012

Asheim, G.B., Mitra, T., Tungodden, B.: Sustainable recursive social welfare functions. Econ. Theory 49, 267-292 (2012)

Banerjee, K.: On the equity-efficiency trade off in aggregating infinite utility streams. Econ. Lett. 93, 63-67 (2006)

Basu, K., Mitra, T.: Aggregating infinite utility streams with intergenerational equity: the impossibility of being Paretian. Econometrica 71, 1557-1563 (2003)

Bossert, W., Sprumont, Y., Suzumura, K.: Ordering infinite utility streams. J. Econ. Theory 135, 579-589 (2007)

Crespo, J., Núñez, C., Rincón-Zapatero, J.P.: On the impossibility of representing infinite utility streams. Econ. Theory 40, 47-56 (2009)

Dalton, H.: The measurement of inequality of incomes. Econ. J. 30, 348-361 (1920)

Dubey, R.S.: Fleurbaey-Michel conjecture on equitable weak Paretian social welfare order. J. Math. Econ. 47, 434-439 (2011) 
Dubey, R.S., Mitra, T.: On equitable social welfare functions satisfying the weak Pareto axiom: a complete characterization. Int. J. Econ. Theory 7, 231-250 (2011)

Fleurbaey, M., Michel, P.: Intertemporal equity and the extension of the Ramsey criterion. J. Math. Econ. 39, 777-802 (2003)

Fleurbaey, M., Tungodden, B.: The tyranny of non-aggregation versus the tyranny of aggregation in social choices: a real dilemma. Econ. Theory 44, 399-414 (2010)

Hara, C., Shinotsuka, T., Suzumura, K., Xu, Y.: On the possibility of continuous Paretian and egalitarian evaluation of infinite utility streams. Mimeo (2006)

Hara, C., Shinotsuka, T., Suzumura, K., Xu, Y.: Continuity and egalitarianism in the evaluation of infinite utility streams. Soc. Choice Welf. 31, 179-191 (2008)

Kolm, S.C.: Multidimensional egalitarianisms. Q. J. Econ. 91, 1-13 (1977)

Lauwers, L.: Ordering infinite utility streams comes at the cost of a non-Ramsey set. J. Math. Econ. 46, 32-37 (2010)

Moulin, H.: Axioms of Cooperative Decision-Making. Cambridge University Press, Cambridge (1991)

Pigou, A.C.: Economics of Welfare, 4th edition, 1932. Macmillan, London (1912)

Sakai, T.: Equitable intergenerational preferences on restricted domains. Soc. Choice Welf. 27, 41-54 (2006)

Sakamoto, N.: Impossibilities of Paretian social welfare functions for infinite utility streams with distributive equity. Hitotsubashi J. Econ. 53, 121-130 (2012)

Zame, W.R.: Can intergenerational equity be operationalized? Theor. Econ. 2, 187-202 (2007) 